\title{
Conformal radii for conformal loop ensembles
}

\author{
Oded Schramm* $\quad$ Scott Sheffield ${ }^{\dagger} \quad$ David B. Wilson
}

\begin{abstract}
The conformal loop ensembles $\mathrm{CLE}_{\kappa}$, defined for $8 / 3 \leq \kappa \leq 8$, are random collections of loops in a planar domain which are conjectured scaling limits of the $O(n)$ loop models. We calculate the distribution of the conformal radii of the nested loops surrounding a deterministic point. Our results agree with predictions made by Cardy and Ziff and by Kenyon and Wilson for the $O(n)$ model. We also compute the expectation dimension of the $\mathrm{CLE}_{\kappa}$ gasket, which consists of points not surrounded by any loop, to be

$$
2-\frac{(8-\kappa)(3 \kappa-8)}{32 \kappa}
$$

which agrees with the fractal dimension given by Duplantier for the $O(n)$ model gasket.
\end{abstract}

\section{Introduction}

The conformal loop ensembles $\mathrm{CLE}_{\kappa}$, defined for all $8 / 3 \leq \kappa \leq 8$, are random collections of loops in a simply connected planar domain $D \varsubsetneqq \mathbb{C}$. They were defined and constructed from branching variants of $\mathrm{SLE}_{\kappa}$ in [She06], where they were conjectured to be the scaling limits of various random loop models from statistical physics, including the so-called $O(n)$ loop models with

$$
n=-2 \cos (4 \pi / \kappa),
$$

see e.g. KN04 for an exposition. This paper is a sequel to [She06]. We will state the results about $\mathrm{CLE}_{\kappa}$ from [She06] that we need for this paper (namely Propositions 1 and 2), but we will not repeat the definition of $\mathrm{CLE}_{\kappa}$ here.

When $8 / 3<\kappa<8, \mathrm{CLE}_{\kappa}$ is almost surely a countably infinite collection of loops. $\mathrm{CLE}_{8}$ is a single space-filling loop almost surely and $\mathrm{CLE}_{8 / 3}$ is almost surely empty. $\mathrm{CLE}_{6}$ is the scaling limit of the cluster boundaries of critical site percolation on the triangular lattice [CN06] Smi01] [CN07]. We will henceforth assume $8 / 3<\kappa<8$.

For each $z \in D$, we inductively define $L_{k}^{z}$ to be the outermost loop surrounding $z$ when the loops $L_{1}^{z}, \ldots, L_{k-1}^{z}$ are removed (provided such a loop exists). For each deterministic $z \in D$, the loops $L_{k}^{z}$ exist for all $k \geq 1$ with probability one. Define $A_{0}^{z}=D$ and let $A_{k}^{z}$ be the component of $D \backslash L_{k}^{z}$ that contains $z$. The conformal gasket is the random closed set $\Gamma$

\footnotetext{
* Microsoft Research.

${ }^{\dagger}$ Courant Institute and Massachusetts Institute of Technology. Partially supported by NSF grant DMS0403182.
} 
consisting of points that are not surrounded by any loop of an instance of $\mathrm{CLE}_{\kappa}$, i.e. the set of points for which $L_{1}^{z}$ does not exist.

If $D$ is a simply connected planar domain and $z \in D$, the conformal radius of $D$ viewed from $z$ is defined to be $\operatorname{CR}(D, z):=\left|g^{\prime}(z)\right|^{-1}$, where $g$ is any conformal map from $D$ to the unit disk $\mathbb{D}$ that sends $z$ to 0 . The following is immediate from the construction in She06]:

Proposition 1. Let $D$ be a simply connected bounded planar domain, and consider a CLE $_{\kappa}$ on $D$ for some $8 / 3<\kappa<8$. Then $\Gamma$ is almost surely the closure of the set of points that lie on an outermost loop (i.e., a loop of the form $L_{1}^{z}$ for some $z$ ). Conditioned on the outermost loops, the law of the remaining loops is given by an independent $\mathrm{CLE}_{\kappa}$ in each component of $D \backslash \Gamma$. For $z \in D$ and $k=1,2,3, \ldots$, define

$$
B_{k}^{z}:=\log \mathrm{CR}\left(A_{k-1}^{z}, z\right)-\log \mathrm{CR}\left(A_{k}^{z}, z\right) .
$$

For any fixed $z$, the $B_{k}^{z}$ 's are i.i.d. random variables.

Various authors in the physics literature have used heuristic arguments (based on the so-called Coulomb gas method) to calculate properties of the scaling limits of statistical physical loop models, including the $O(n)$ models, based on certain conformal invariance hypotheses of these limits. Although the scaling limits of the $O(n)$ models have not been shown to exist, there is strong evidence that if they do exist they must be CLE $_{\kappa}$. (For example, there is heuristic evidence that any scaling limit of the $O(n)$ models should be in some sense conformally invariant; it is shown in [She06] SW08b] SW08a] that any random loop ensemble satisfying certain hypotheses including conformal invariance and a Markovtype property must be a $\mathrm{CLE}_{\kappa}$.) It is therefore natural to interpret these calculations as predictions about the behavior of the $\mathrm{CLE}_{\kappa}$.

Cardy and Ziff CZ03 predicted and experimentally verified the expected number of loops surrounding a point in the $O(n)$ model, which, in light of (1), may be interpreted as a prediction of the expectation of $B_{z}^{k}$ :

$$
\frac{1}{\mathbb{E}\left[B_{k}^{z}\right]}=\frac{(\kappa / 4-1) \cot (\pi(1-4 / \kappa))}{\pi} .
$$

Kenyon and Wilson [KW04] went further and predicted the distribution of $B_{k}^{z}$, giving its moment generating function

$$
\mathbb{E}\left[\exp \left(\lambda B_{k}^{z}\right)\right]=\frac{-\cos (4 \pi / \kappa)}{\cos \left(\pi \sqrt{(1-4 / \kappa)^{2}+8 \lambda / \kappa}\right)}
$$

for $\lambda$ satisfying $\operatorname{Re} \lambda<1-\frac{2}{\kappa}-\frac{3 \kappa}{32}$, and density function

$$
\frac{d}{d x} \operatorname{Pr}\left[B_{k}^{z}<x\right]=\frac{-\kappa \cos (4 \pi / \kappa)}{4 \pi} \sum_{j=0}^{\infty}(-1)^{j}(j+1 / 2) \exp \left[-\frac{(j+1 / 2)^{2}-(1-4 / \kappa)^{2}}{8 / \kappa} x\right] .
$$

The main result of this paper is Theorem 1, which confirms these predictions. In the special case $\kappa=6$, this prediction for the law of $B_{k}^{z}$ was independently confirmed by Dubédat Dub05. 
Theorem 1. Let $f_{\kappa}$ denote the density function for the first time that a standard Brownian motion started at 0 exits the interval $(-2 \pi / \sqrt{\kappa}, 2 \pi / \sqrt{\kappa})$. Then for $8 / 3<\kappa<8$, the density function for $B_{k}^{z}$ is

$$
\frac{d}{d x} \operatorname{Pr}\left[B_{k}^{z}<x\right]=-f_{\kappa}(x) \cos (4 \pi / \kappa) \exp \left[\frac{(\kappa-4)^{2}}{8 \kappa} x\right] .
$$

The equivalence of the formulations (4) and (5), and the fact that they imply (3), follows from a calculation of Ciesielski and Taylor, who showed that the exit-time distribution of a Brownian motion from the center of a 1-dimensional ball of radius $r$ has a moment generating function of $1 / \cos \sqrt{2 r^{2} \lambda}$, and who gave two series expansions (one in powers of $e^{-x}$ and the other in powers of $e^{-1 / x}$ ) for its density function [CT62, Theorem 2 and eq. 2.22] (see also [BS02, eqs. 1.3.0.1 and 1.3.0.2]). Since the Fourier transform is invertible on $L^{2}(\mathbb{R})$, the equivalence of (3) and (44) follows by considering the moment generating function restricted to the imaginary line $\operatorname{Re} \lambda=0$.

Duplantier [Dup90 predicted the fractal dimension of the gasket $\Gamma$ associated with the $O(n)$ model to be

$$
\frac{3 \kappa}{32}+1+\frac{2}{\kappa},
$$

where as usual $n=-2 \cos (4 \pi / \kappa)$. We partially confirm this prediction by giving the expectation dimension of the gasket associated with $\mathrm{CLE}_{\kappa}$. The expectation dimension of a random bounded set $A$ is defined to be

$$
D_{\mathbb{E}}(A)=\lim _{\varepsilon \rightarrow 0} \frac{\log \mathbb{E}[\text { minimal number of balls of radius } \varepsilon \text { required to cover } A]}{|\log \varepsilon|},
$$

provided the limit exists. The expectation dimension upper bounds the Hausdorff dimension.

Theorem 2. Let $\Gamma$ be the gasket of a $\mathrm{CLE}_{\kappa}$ in the unit disk with $\kappa \in(8 / 3,8)$. Then

$$
\mathbb{E}[\text { minimal number of balls of radius } \varepsilon \text { required to cover } \Gamma] \asymp\left(\frac{1}{\varepsilon}\right)^{\frac{3 \kappa}{32}+1+\frac{2}{\kappa}} \text {. }
$$

In particular, the expectation dimension of the gasket $\Gamma$ is $\frac{3 \kappa}{32}+1+\frac{2}{\kappa}$.

Here, $\asymp$ denotes equivalence up to multiplicative constants. Lawler, Schramm, and Werner [LSW02] studied the percolation gasket (associated with $\mathrm{CLE}_{6}$ ), effectively proving Theorem 2 in the case $\kappa=6$. More generally, they studied how long it takes for a radial $\mathrm{SLE}_{\kappa}$ to surround the origin when $\kappa>4$, and their results implicitly imply Theorem 2 when $\kappa>4$; see the remark in Section 2.1 for further discussion.

We conclude our introduction by noting that the gasket dimension described above plays an important role in the physics literature, where it is related (at least heuristically) to the exponents of magnetization and multipoint correlation functions in critical lattice models. We briefly describe this connection in the case of the $q$-state Potts model on the square lattice. More details and references are found in [She06], [Car07], Gri06].

A sample from the $q$-state Potts model on a connected planar graph $G$ is a random function $\sigma: V \rightarrow\{1,2, \ldots, q\}$, where $V$ is the set of vertices of $G$ and the image values 
$1,2, \ldots, q$ are often called spins. If the boundary vertices (those on the boundary of the unbounded face) of $G$ are all assigned a particular value (say $b$ ), then using the standard FK random cluster decomposition [FK72], one may construct a sample from the Potts model as follows:

1. Sample a random subgraph $G^{\prime}$ of $G$ containing all boundary edges (edges on the boundary of the unbounded face), with probability proportional to

$$
q^{\# \text { components of } G^{\prime}}\left(\frac{p}{1-p}\right)^{\# \text { edges of } G^{\prime}},
$$

where $0<p<1$ is a parameter. The law of $G^{\prime}$ is called the FK random cluster model with parameters $p$ and $q$. Call the component of $G^{\prime}$ which contains the boundary vertices of $G$ the FK gasket.

2. Set $\sigma(v)=b$ for each $v$ in the FK gasket, and independently assign one of the $q$ states uniformly at random to each of the remaining connected components of $G^{\prime}$ (assigning all vertices in that component the corresponding state).

The "magnetization" at an interior vertex $v$ of $G$ (i.e., the probability that $\sigma(v)=j$ minus $1 / q$ ) is proportional to the probability that $v$ is in the FK gasket. Given distinct vertices $v$ and $w$, the covariance of $\sigma(v)$ and $\sigma(w)$ is proportional to the probability that both $v$ and $w$ lie in the same component of $G^{\prime}$.

We now restrict to the case in which $G$ is a finite piece of the square grid in the plane and the parameter $p$ satisfies $p /(1-p)=\sqrt{q}$. (With this choice of $p$, the FK model is self-dual and believed to be critical, see e.g., [Gri06, Chapter 6].)

It is shown in [She06] that if $q \leq 4$ and certain other hypotheses including conformal invariance hold, then the scaling limit of the set of boundaries between clusters and dual clusters in the critical FK random cluster models discussed above must be given by CLE $_{\kappa}$ for the $\kappa$ satisfying $q=4 \cos ^{2}(4 \pi / \kappa)$ and $4 \leq \kappa \leq 8$. Assuming these hypotheses, the scaling limit of the discrete gasket is precisely the continuum $\mathrm{CLE}_{\kappa}$ gasket.

A heuristic ansatz is that the law of the critical FK gasket should have similar properties as the law of the set of squares in a fine grid which intersect the continuum gasket. If this heuristic holds, then when $G$ is a bounded domain intersected with a square grid with spacing $\varepsilon$, the magnetization at a vertex $v$ of macroscopic distance from the boundary in the discrete model will be on the order of $\varepsilon^{2-d}$, where $d$ is the limiting expectation dimension of the continuum gasket. Similarly, the covariance between $\sigma(v)$ and $\sigma(w)$, for two macroscopically separated vertices $v$ and $w$, should be on the order of $\varepsilon^{2(2-d)}$ (since in the continuum model, the set of pairs $v$ and $w$ which lie in the same continuum spin cluster has dimension 2d; see [She06]).

\section{Diffusions and martingales}

\subsection{Reduction to a diffusion problem}

Let $B_{t}:[0, \infty) \rightarrow \mathbb{R}$ be a standard Brownian motion and let $\theta_{t}:[0, \infty) \rightarrow[0,2 \pi]$ be a random continuous process on the interval $[0,2 \pi]$ that is instantaneously reflecting at its 
endpoints (i.e., the set $\left\{t: \theta_{t} \in\{0,2 \pi\}\right\}$ has Lebesgue measure zero almost surely) and evolves according to the SDE

$$
d \theta_{t}=\frac{\kappa-4}{2} \cot \left(\theta_{t} / 2\right) d t+\sqrt{\kappa} d B_{t}
$$

on each interval of time for which $\theta_{t} \notin\{0,2 \pi\}$. In other words, $\theta_{t}$ is a random continuous process adapted to the filtration of $B_{t}$ which almost surely satisfies

$$
\frac{\partial}{\partial t}\left(\theta_{t}-\sqrt{\kappa} B_{t}\right)=\frac{\kappa-4}{2} \cot \left(\theta_{t} / 2\right)
$$

for all $t$ for which the right hand side is well defined. The law of this process is uniquely determined by $\theta_{0}$ [She06], and we also have the following from [She06]:

Proposition 2. When $8 / 3<\kappa<8$, the law of $B_{k}^{z}$ is the same as the law of $\inf \left\{t: \theta_{t}=2 \pi\right\}$ for the diffusion (6) started at $\theta_{0}=0$.

It is convenient to lift the process $\theta_{t}$ so that, rather than taking values in $[0,2 \pi]$, it takes values in all of $\mathbb{R}$. Let $R: \mathbb{R} \rightarrow[0,2 \pi]$ be the piecewise affine map for which $R(x)=|x|$ when $x \in[-2 \pi, 2 \pi]$ and $R(4 \pi+x)=R(x)$ for all $x$. Given $\theta_{t}$, we can generate a continuous process $\tilde{\theta}_{t}$ with $R\left(\tilde{\theta}_{t}\right)=\theta_{t}$ in such a way that for each component $\left(t_{1}, t_{2}\right)$ of the set $\{t$ : $\left.\theta_{t} \notin 2 \pi \mathbb{Z}\right\}$, we independently toss a fair coin to decide whether $\tilde{\theta}_{t}>\tilde{\theta}_{t_{1}}$ or $\tilde{\theta}_{t}<\tilde{\theta}_{t_{1}}$ on that component. The $\theta_{t}$ together with these coin tosses (for each interval of $\left\{t: \theta_{t} \notin 2 \pi \mathbb{Z}\right\}$ ) determine $\tilde{\theta}_{t}$ uniquely.

This $\tilde{\theta}_{t}$ is still a solution to (6) provided we modify $B_{t}$ in such a way that $d B_{t}$ is replaced with $-d B_{t}$ on those intervals for which $d \tilde{\theta}_{t}=-d \theta_{t}$. (This modification does not change the law of $B_{t}$.) In the remainder of the text, we will drop the $\tilde{\theta}_{t}$ notation and write $\theta_{t}$ for the lifted process on $\mathbb{R}$.

Remark: A very similar diffusion process was studied by Lawler, Schramm, and Werner [LSW02, namely

$$
d \theta_{t}=\cot \left(\theta_{t} / 2\right) d t+\sqrt{\kappa} d B_{t}
$$

which is the same as ([6) but without the factor of $(\kappa-4) / 2$, and they too studied the time for the diffusion to reach $\theta_{t}=2 \pi$ when started at $\theta_{0}=0$. Diffusions 6 and 7 are identical when $\kappa=6$, and Diffusion 6 (for $4<\kappa<8$ ) is given by Diffusion 7 (for $4<\kappa<\infty$ ) upon substituting $\kappa \rightarrow(2 \kappa) /(\kappa-4)$ and scaling time by $t \rightarrow t(\kappa-4) / 2$. However, Diffusion 6 is a more singular Bessel-type process when $\kappa \leq 4$, requiring additional technical analysis to deal with the times at which process is at 0 (see e.g. Lemma 3). Furthermore, only the large-time asymptotic decay rate of the hitting time distribution (used in the proof of Theorem 2) is given in [LSW02], and additional effort is required to obtain the precise hitting time distribution provided in Theorem 1.

\subsection{Local martingales}

Recall the hypergeometric function defined by

$$
F(a, b ; c ; z)=\sum_{n=0}^{\infty} \frac{(a)_{n}(b)_{n}}{(c)_{n} n !} z^{n},
$$


where $a, b, c \in \mathbb{C}$ are parameters, $c \notin-\mathbb{N}$ (where $\mathbb{N}=\{0,1,2, \ldots\}$ ), and $(\ell)_{n}$ denotes $\ell(\ell+1) \cdots(\ell+n-1)$. This definition holds for $z \in \mathbb{C}$ when $|z|<1$, and it may be defined by analytic continuation elsewhere (though it is then not always single-valued). We define for $\lambda \in \mathbb{C}$

$$
\begin{aligned}
& M_{\kappa, \lambda}^{e}(\theta)=F\left(1-\frac{4}{\kappa}+\sqrt{\left(1-\frac{4}{\kappa}\right)^{2}+\frac{8 \lambda}{\kappa}}, 1-\frac{4}{\kappa}-\sqrt{\left(1-\frac{4}{\kappa}\right)^{2}+\frac{8 \lambda}{\kappa}} ; \frac{3}{2}-\frac{4}{\kappa} ; \sin ^{2} \frac{\theta}{4}\right) \\
& M_{\kappa, \lambda}^{o}(\theta)=F\left(1-\frac{2}{\kappa}+\sqrt{\left(\frac{1}{2}-\frac{2}{\kappa}\right)^{2}+\frac{2 \lambda}{\kappa}}, 1-\frac{2}{\kappa}-\sqrt{\left(\frac{1}{2}-\frac{2}{\kappa}\right)^{2}+\frac{2 \lambda}{\kappa}} ; \frac{3}{2} ; \cos ^{2} \frac{\theta}{2}\right) \cos \frac{\theta}{2}
\end{aligned}
$$

(where the formula for $M_{\kappa, \lambda}^{e}(\theta)$ makes sense whenever $\kappa \neq \frac{8}{3}, \frac{8}{5}, \frac{8}{7}, \ldots$ ). There is some ambiguity in the choice of square root, but since $F(b, a ; c ; z)=F(a, b ; c ; z)$, as long as the same choice of square root is made for both occurences, there is no ambiguity in these definitions.

Lemma 1. For the diffusion (6) with $\kappa>0$, let $T$ be the first time at which $\theta_{t} \in 2 \pi \mathbb{Z}$, and let $\bar{t}=\min (t, T)$. For any $\lambda \in \mathbb{C}$, both $\exp [\lambda \bar{t}] M_{\kappa, \lambda}^{e}\left(\theta_{\bar{t}}\right)$ and $\exp [\lambda \bar{t}] M_{\kappa, \lambda}^{o}\left(\theta_{\bar{t}}\right)$ are local martingales parameterized by $t$.

Proof. Given these formulas, in principle it is straightforward to verify that the $d t$ term of the Itô expansion of

$$
d\left[e^{\lambda t} M_{\kappa, \lambda}^{e \mid o}\left(\theta_{t}\right)\right]
$$

is equal to zero (where $e \mid o$ is either $e$ or $o$ ). This term can be expressed as

$$
e^{\lambda t}\left[\lambda M_{\kappa, \lambda}^{e \mid o}\left(\theta_{t}\right)+\left(M_{\kappa, \lambda}^{e \mid o}\right)^{\prime}\left(\theta_{t}\right) \frac{\kappa-4}{2} \cot \left(\theta_{t} / 2\right)+\frac{\kappa}{2}\left(M_{\kappa, \lambda}^{e \mid o}\right)^{\prime \prime}\left(\theta_{t}\right)\right] d t
$$

Since Mathematica does not simplify this to zero, we show how to do this for $M_{\kappa, \lambda}^{e}$; the case for $M_{\kappa, \lambda}^{o}$ is similar.

We abbreviate $M_{\kappa, \lambda}^{e}$ with $M$, let $F$ denote the hypergeometric function in the definition of $M_{\kappa, \lambda}^{e}$, let $a, b$, and $c$ denote the parameters of $F$ in $M$, and change variables to $y=y(\theta)=$ $\sin ^{2}(\theta / 4)=(1-\cos (\theta / 2)) / 2$ :

$$
\begin{aligned}
M(\theta) & =F(y(\theta)) \\
M^{\prime}(\theta) & =\frac{1}{4} F^{\prime}(y(\theta)) \sin (\theta / 2) \\
M^{\prime \prime}(\theta) & =F^{\prime \prime}(y(\theta)) \frac{1}{16} \sin ^{2}(\theta / 2)+F^{\prime}(y(\theta)) \frac{1}{8} \cos (\theta / 2) \\
& =F^{\prime \prime}(y) \frac{y-y^{2}}{4}+F^{\prime}(y) \frac{\frac{1}{2}-y}{4},
\end{aligned}
$$


so that

$$
\begin{array}{r}
e^{\lambda t}\left[\lambda F(y)-\frac{\kappa-4}{4} F^{\prime}(y)\left(y-\frac{1}{2}\right)+\frac{\kappa}{8} F^{\prime \prime}(y)\left(y-y^{2}\right)+\frac{\kappa}{8} F^{\prime}(y)\left(\frac{1}{2}-y\right)\right] \\
=e^{\lambda t}\left[\lambda F(y)+(1-3 \kappa / 8) F^{\prime}(y)\left(y-\frac{1}{2}\right)+\frac{\kappa}{8} F^{\prime \prime}(y)\left(y-y^{2}\right)\right] \\
=e^{\lambda t} \sum_{n=0}^{\infty} y^{n} \frac{(a)_{n}(b)_{n}}{(c)_{n} n !} \underbrace{\left[\begin{array}{c}
\lambda+(1-3 \kappa / 8)\left(n-\frac{1}{2} \frac{(a+n)(b+n)}{c+n}\right)+ \\
+\frac{\kappa}{8}\left(\frac{(a+n)(b+n)}{c+n} n-n(n-1)\right)
\end{array}\right]}_{E_{n} /(c+n)}
\end{array}
$$

and we define $E_{n}$ to be $c+n$ times the expression in brackets. (Note that indeed $c \notin-\mathbb{N}$.) We may write $E_{n}$ as a polynomial in $n$ :

$$
\begin{aligned}
E_{n}= & {[(1-3 \kappa / 8)(1-1 / 2)+(\kappa / 8)(1-c+a+b)] \times n^{2}+} \\
& {[\lambda+(1-3 \kappa / 8)(c-a / 2-b / 2)+(\kappa / 8)(c+a b)] \times n+} \\
& {[c \lambda-(1-3 \kappa / 8) a b / 2] . }
\end{aligned}
$$

By our choices of $a, b$ and $c, E_{n}=0$ for each $n$, which proves the claim for $M_{\kappa, \lambda}^{e}$.

\section{$2.3 \quad$ Expected first hitting of $2 \pi \mathbb{Z}$}

In this subsection we obtain asymptotics for the function

$$
L(\theta):=\mathbb{E}\left[\theta_{T} \mid \theta_{0}=\theta\right],
$$

where $\theta_{t}$ is the diffusion (6) and $T$ is the first time $t \geq 0$ at which $\theta_{t} \in 2 \pi \mathbb{Z}$. (Recall that $T$ is finite a.s. when $\kappa<8$.) Whenever $\theta \in 2 \pi \mathbb{Z}$, trivially $L(\theta)=\theta$.

Lemma 2. For the diffusion (6) with $8 / 3<\kappa<8, L\left(\theta_{t}\right)$ is a martingale.

Proof. Since $L(\theta)$ is defined in terms of expected values, $L\left(\theta_{t}\right)$ is a local martingale whenever $\theta_{t} \notin 2 \pi \mathbb{Z}$, and the stopped process $L\left(\theta_{\min (t, T)}\right)$ is a martingale. Since the diffusion behaves symmetrically around the points $2 \pi \mathbb{Z}$ and the number of intervals of $\mathbb{R} \backslash 2 \pi \mathbb{Z}$ crossed before some deterministic time has exponentially decaying tails (which implies integrability), $L\left(\theta_{t}\right)$ is a martingale.

Next, we express $L\left(\theta_{t}\right)$ in terms of the $\lambda=0$ case of the local martingales $\exp [\lambda t] M_{\kappa, \lambda}^{e}\left(\theta_{t}\right)$ and $\exp [\lambda t] M_{\kappa, \lambda}^{o}\left(\theta_{t}\right)$. Because $M_{\kappa, 0}^{e}(\theta)=1$, this local martingale is uninformative, but

$$
M_{\kappa, 0}^{o}(\theta)=F\left(\frac{3}{2}-\frac{4}{\kappa}, \frac{1}{2} ; \frac{3}{2} ; \cos ^{2} \frac{\theta}{2}\right) \cos \frac{\theta}{2} .
$$

We have $M_{\kappa, 0}^{o}(0)=-M_{\kappa, 0}^{o}(2 \pi)=\sqrt{\pi} \Gamma(4 / \kappa-1 / 2) /(2 \Gamma(4 / \kappa))$, and since $M_{\kappa, 0}^{o}$ is bounded (when $\kappa \neq 8$ ), the stopped process $M_{\kappa, 0}^{o}\left(\theta_{\min (t, T)}\right)$ is also a martingale. This determines $L$, namely,

$$
L(\theta)=\pi-\frac{2 \sqrt{\pi} \Gamma\left(\frac{4}{\kappa}\right)}{\Gamma\left(\frac{4}{\kappa}-\frac{1}{2}\right)} F\left(\frac{3}{2}-\frac{4}{\kappa}, \frac{1}{2} ; \frac{3}{2} ; \cos ^{2} \frac{\theta}{2}\right) \cos \frac{\theta}{2}, \quad \theta \in[0,2 \pi]
$$


and $L(\theta+2 \pi)=L(\theta)+2 \pi$. (It is also possible to derive (8) from the formula for $\operatorname{Pr}$ [SLE trace passes to left of $x+i y$ ] [Sch01] after applying a Möbius transformation and suitable hypergeometric identities.)

We wish to understand the behavior of $L$ near the points in $2 \pi \mathbb{Z}$, and to this end we use the formula (see [EMOT53, pg. 108, Eq. 2.10.1])

$$
\begin{aligned}
F(a, b ; c ; z)= & \frac{\Gamma(c) \Gamma(c-a-b)}{\Gamma(c-a) \Gamma(c-b)} F(a, b ; a+b-c+1 ; 1-z)+ \\
& \quad+\frac{\Gamma(c) \Gamma(a+b-c)}{\Gamma(a) \Gamma(b)}(1-z)^{c-a-b} F(c-a, c-b ; c-a-b+1 ; 1-z)
\end{aligned}
$$

which is valid when $1-c, b-a$, and $c-b-a$ are not integers and $|\arg (1-z)|<\pi$. In our case the nonintegrality condition is satisfied whenever $8 / \kappa \notin \mathbb{N}$. For the range of $\kappa$ that we are interested in, this rules out $\kappa=4$, for which we already know $L(\theta)=\theta$, and therefore do not need asymptotics. The endpoints of the range, $\kappa=8 / 3$ and $\kappa=8$ are also ruled out, but for the remaining $\kappa$ 's we have

$$
\begin{aligned}
F\left(\frac{3}{2}-\frac{4}{\kappa}, \frac{1}{2} ; \frac{3}{2} ; \cos ^{2} \frac{\theta}{2}\right) & =\frac{\Gamma\left(\frac{3}{2}\right) \Gamma\left(\frac{4}{\kappa}-\frac{1}{2}\right)}{\Gamma\left(\frac{4}{\kappa}\right) \Gamma(1)} F\left(\frac{3}{2}-\frac{4}{\kappa}, \frac{1}{2} ; \frac{3}{2}-\frac{4}{\kappa} ; \sin ^{2} \frac{\theta}{2}\right)+ \\
& +\frac{\Gamma\left(\frac{3}{2}\right) \Gamma\left(\frac{1}{2}-\frac{4}{\kappa}\right)}{\Gamma\left(\frac{3}{2}-\frac{4}{\kappa}\right) \Gamma\left(\frac{1}{2}\right)}\left|\sin \frac{\theta}{2}\right|^{\frac{8}{\kappa}-1} F\left(\frac{4}{\kappa}, 1 ; \frac{4}{\kappa}+\frac{1}{2} ; \sin ^{2} \frac{\theta}{2}\right) \\
& =\frac{\sqrt{\pi} \Gamma\left(\frac{4}{\kappa}-\frac{1}{2}\right)}{2 \Gamma\left(\frac{4}{\kappa}\right)} \frac{1}{\left|\cos \frac{\theta}{2}\right|}+\frac{\Gamma\left(\frac{1}{2}-\frac{4}{\kappa}\right)}{2 \Gamma\left(\frac{3}{2}-\frac{4}{\kappa}\right)}\left|\sin \frac{\theta}{2}\right|^{\frac{8}{\kappa}-1} F\left(\frac{4}{\kappa}, 1 ; \frac{4}{\kappa}+\frac{1}{2} ; \sin ^{2} \frac{\theta}{2}\right),
\end{aligned}
$$

and by (8)

$$
L(\theta)=c_{\kappa}\left(\sin \frac{\theta}{2}\right)^{\frac{8}{\kappa}-1} F\left(\frac{4}{\kappa}, 1 ; \frac{4}{\kappa}+\frac{1}{2} ; \sin ^{2} \frac{\theta}{2}\right) \cos \frac{\theta}{2}, \quad \theta \in(0, \pi) .
$$

for some constant $c_{\kappa}>0$. (One can use the Legendre duplication formula to show that $c_{\kappa}=2^{8 / \kappa-1} \Gamma\left(\frac{4}{\kappa}\right)^{2} / \Gamma\left(\frac{8}{\kappa}\right)$, but we do not need this.) Since $L(-\theta)=-L(\theta)$, we conclude that

$$
L(\theta)=A_{0}\left(\theta^{2}\right)|\theta|^{8 / \kappa} / \theta, \quad \theta \in(-\pi, \pi),
$$

where $A_{0}$ is a analytic function (depending on $\kappa$ ) satisfying $A_{0}(0)>0$. This implies

$$
\theta^{2}=A\left(|L(\theta)|^{2 \kappa /(8-\kappa)}\right)
$$

near $\theta=0$, for some analytic $A$.

\subsection{Starting at $\theta_{0}=0$}

We will eventually need to start the diffusion at $\theta_{0}=0$, but Lemma 1 only covers what happens up until the first time that $\theta_{t} \in 2 \pi \mathbb{Z}$. In this subsection we show

Lemma 3. For the diffusion (6) with $8 / 3<\kappa<8$, let $T$ be the first time at which $\theta_{t} \in$ $2 \pi \mathbb{Z} \backslash 4 \pi \mathbb{Z}$, and let $\bar{t}=\min (t, T)$. For any $\lambda \in \mathbb{C}$, the process $\exp [\lambda \bar{t}] M_{\kappa, \lambda}^{e}\left(\theta_{\bar{t}}\right)$ is a martingale. 
Proof. Let $M$ abbreviate $M_{\kappa, \lambda}^{e}$, and let us assume without loss of generality that $-2 \pi<$ $\theta_{0}<2 \pi$. Let us define $\omega_{t}=L\left(\theta_{t}\right)$, which is a martingale and may be interpreted as a time-changed Brownian motion. We wish to argue that $e^{\lambda \bar{t}} M\left(L^{-1}\left(\omega_{\bar{t}}\right)\right)=e^{\lambda \bar{t}} M\left(\theta_{\bar{t}}\right)$ is a local martingale. (The definition of $L$ implies that it is strictly monotone, and hence $L^{-1}$ is well defined.) Note that by Lemma 1 it is a local martingale when $\theta_{\bar{t}} \notin 2 \pi \mathbb{Z}$. To extend this to a neighborhood of $\theta_{\bar{t}}=0$, one could try to use Itô's formula. To do this, it would be necessary that $f:=M \circ L^{-1}$ be twice differentiable. We have $M(\theta)=A_{1}\left(\theta^{2}\right)$ in $(-2 \pi, 2 \pi)$, where $A_{1}$ is analytic. Consequently, (10) gives for $8 / 3<\kappa<8$ and for $\omega$ in a neighborhood of 0 ,

$$
f(\omega)=A_{2}\left(|\omega|^{2 \kappa /(8-\kappa)}\right)
$$

for some analytic $A_{2}$. Though this is not necessary for the proof, one can check that $A_{2}^{\prime}(0) \neq 0$ and therefore $f^{\prime \prime}(0)$ is not finite when $\kappa<4$.

To circumvent the problem of $f=M \circ L^{-1}$ not being twice differentiable, we use the Itô-Tanaka Theorem ([RY99, Theorem 1.5 on pg. 223]). The exponent $\frac{2 \kappa}{8-\kappa}$ in (11) ranges from 1 to $\infty$ as $\kappa$ ranges from $8 / 3$ to 8 . In particular, $f^{\prime}(0)=0$ and $f^{\prime}$ is continuous near 0 . Since $A_{2}$ is analytic, near 0 the function $f$ may be expressed as the difference of two convex functions, namely, $f(\omega)=(f(\omega)-f(0)) 1_{\omega \geq 0}+(f(\omega)-f(0)) 1_{\omega \leq 0}+f(0)$. Therefore, we may apply the Itô-Tanaka Theorem to conclude that $e^{\lambda \bar{t}} f\left(\omega_{\bar{t}}\right)=e^{\lambda \bar{t}} M\left(\theta_{\bar{t}}\right)$ is a local martingale also when $\theta_{\bar{t}}$ is near zero.

Now, the hypergeometric function $F$ satisfies

$$
F(a, b ; c ; 1)=\frac{\Gamma(c) \Gamma(c-a-b)}{\Gamma(c-a) \Gamma(c-b)}
$$

provided $-c \notin \mathbb{N}$ and $\operatorname{Re} c>\operatorname{Re}(a+b)$ (see e.g. [EMOT53, pg. 104 eq. 46]). Therefore, $M( \pm 2 \pi)$ is finite. Thus, $e^{\lambda \bar{t}} M\left(\theta_{\bar{t}}\right)$ is bounded for bounded $t$, and we may conclude that it is a martingale.

For future reference, we now calculate $M_{\kappa, \lambda}^{e}( \pm 2 \pi)$. Observe that the parameters $a, b, c$ of the hypergeometric function in the definition of $M_{\kappa, \lambda}^{e}$ satisfy $2 c-a-b=1$. Consequently, the identity $\Gamma(z) \Gamma(1-z)=\pi / \sin (\pi z)$ and (12) give

$$
M_{\kappa, \lambda}^{e}( \pm 2 \pi)=\frac{\sin \left(\frac{\pi}{2}-\pi \sqrt{\left(1-\frac{4}{\kappa}\right)^{2}+\frac{8 \lambda}{\kappa}}\right)}{\sin (3 \pi / 2-4 \pi / \kappa)}=\frac{\cos \left(\pi \sqrt{(1-4 / \kappa)^{2}+8 \lambda / \kappa}\right)}{\cos (\pi(1-4 / \kappa))}
$$

\section{Proofs of main results}

We now restate and prove Theorem 1,

Theorem 3. Suppose the diffusion process (6) (with $8 / 3<\kappa<8$ ) is started at $\theta_{0}=0$, and $T$ is the first time at which $\theta_{t}= \pm 2 \pi$. If $\operatorname{Re} \lambda \leq 0$, then

$$
\mathbb{E}\left[e^{\lambda T} \mid \theta_{0}=0\right]=\frac{\cos (\pi(1-4 / \kappa))}{\cos \left(\pi \sqrt{(1-4 / \kappa)^{2}+8 \lambda / \kappa}\right)}
$$


(This is equivalent to Theorem 1 by Proposition 2 and the remarks following the statement of Theorem 1.)

Proof. Since $M_{\kappa, \lambda}^{e}\left(\theta_{T}\right)=M_{\kappa, \lambda}^{e}( \pm 2 \pi)=M_{\kappa, \lambda}^{e}(2 \pi)$ a.s. and $\exp [\lambda \bar{t}] M_{\kappa, \lambda}^{e}\left(\theta_{\bar{t}}\right)$ is a martingale, the optional sampling theorem gives

$$
M_{\kappa, \lambda}^{e}(2 \pi) \mathbb{E}\left[e^{\lambda T} \mid \theta_{0}=0\right]=\mathbb{E}\left[e^{\lambda T} M_{\kappa, \lambda}^{e}\left(\theta_{T}\right) \mid \theta_{0}=0\right]=M_{\kappa, \lambda}^{e}(0)=1,
$$

and the proof is completed by appeal to (13).

Proof of Theorem 2. Fix some $\varepsilon>0$ and let $z \in \mathbb{D}$. Set $r_{0}:=1-|z|, r_{1}:=\operatorname{dist}\left(z, L_{1}^{z}\right)$, and suppose that $\varepsilon<r_{0}$. We seek to estimate the probability that the open disk of radius $\varepsilon$ about $z$ intersects the gasket; that is, the probability that $r_{1}<\varepsilon$. By the Koebe $1 / 4$ theorem, $r_{1} \leq \mathrm{CR}\left(D_{1}, z\right) \leq 4 r_{1}$. Likewise, $r_{0} \leq \mathrm{CR}(\mathbb{D}, z) \leq 4 r_{0}$. Thus, $B_{1}^{z}=\log \mathrm{CR}(\mathbb{D}, z)-$ $\log \mathrm{CR}\left(D_{1}, z\right)=\log \left(r_{0} / r_{1}\right)+O(1)$. Referring to the density function of $B_{k}^{z}$ (4), we see that

$$
\operatorname{Pr}\left[r_{1}<\varepsilon\right] \asymp \exp \left[-\alpha \log \left(r_{0} / \varepsilon\right)\right]=\left(\varepsilon / r_{0}\right)^{\alpha}
$$

where

$$
\alpha=\frac{1 / 4-(1-4 / \kappa)^{2}}{8 / \kappa}=-\frac{3 \kappa}{32}+1-\frac{2}{\kappa}=\frac{(8-\kappa)(3 \kappa-8)}{32 \kappa} .
$$

For each $j=1, \ldots,\lceil 1 / \varepsilon\rceil$, we may cover the annulus $\{z:(j-1) \varepsilon \leq 1-|z| \leq j \varepsilon\}$ by $O(1 / \varepsilon)$ disks of radius $\varepsilon$. The total expected number of these disks that intersect the gasket is at most

$$
\sum_{j=1}^{\lceil 1 / \varepsilon\rceil} O(1 / \varepsilon) \times O(\varepsilon /(j \varepsilon))^{\alpha}=O\left(\varepsilon^{\alpha-2}\right) .
$$

(Here we made use of the fact that $\alpha<1$.) Thus on average $O\left(\varepsilon^{\alpha-2}\right)$ disks of radius $\varepsilon$ suffice to cover the gasket.

On the other hand, we may pack into $\mathbb{D}$ at least $\Theta\left(1 / \varepsilon^{2}\right)$ points so that every two of them are more than distance $4 \varepsilon$ apart, and each of them is at least distance $1 / 2$ from the boundary. For each such point $z$ there is a $\Theta\left(\varepsilon^{\alpha}\right)$ chance that the disk or radius $\varepsilon$ centered at $z$ is not surrounded by a loop, i.e., that that the gasket $\Gamma$ contains a point $z^{\prime}$ that is within distance $\varepsilon$ of $z$. Since the points $z$ are sufficiently far apart, the points $z^{\prime}$ must be covered by distinct disks in any covering of $\Gamma$ by disks of radius $\varepsilon$. Thus the expected number of disks of radius $\varepsilon$ required to cover the gasket $\Gamma$ is at least $\Theta\left(\varepsilon^{\alpha-2}\right)$.

\section{Open problems}

Kenyon and Wilson [KW04] also predicted the large- $k$ limiting distribution of another quantity, the "electrical thickness" of the loops $L_{k}^{z}$ when $k \rightarrow \infty$. The electrical thickness of a loop compares the conformal radius of the loop to the conformal radius of the image of the loop under the map $m(w)=1 /(w-z)$, and more precisely it is

$$
\vartheta_{z}\left(L_{k}^{z}\right)=-\log \mathrm{CR}\left(L_{k}^{z}, z\right)-\log \mathrm{CR}\left(m\left(L_{k}^{z}\right), z\right) .
$$


Kenyon and Wilson [KW04] predicted that the large- $k$ moment generating function of $\vartheta_{z}\left(L_{k}^{z}\right)$ is

$$
\lim _{k \rightarrow \infty} \mathbb{E}\left[\exp \left(\lambda \vartheta_{z}\left(L_{k}^{z}\right)\right)\right]=\frac{\sin (\pi(1-4 / \kappa))}{\pi(1-4 / \kappa)} \frac{\pi \sqrt{(1-4 / \kappa)^{2}+8 \lambda / \kappa}}{\sin \left(\pi \sqrt{(1-4 / \kappa)^{2}+8 \lambda / \kappa}\right)},
$$

or equivalently that the limiting probability density function is given by the density function of the exit time of a standard Brownian excursion started in the middle of the interval $(-2 \pi / \sqrt{\kappa}, 2 \pi / \sqrt{\kappa})$, reweighted by a factor of const $\times \exp \left[(\kappa-4)^{2} x /(8 \kappa)\right]$. (This equivalence follows from [BS02, eq. 5.3.0.1].) Recall that the density function of $B_{k}^{z}$ is given by the density function of the exit time of a standard Brownian motion started in the middle of the interval $(-2 \pi / \sqrt{\kappa}, 2 \pi / \sqrt{\kappa})$, also reweighted by a factor of const $\times \exp \left[(\kappa-4)^{2} x /(8 \kappa)\right]$. These forms are highly suggestive, but currently we do not know how to calculate the electrical thickness using $\mathrm{CLE}_{\kappa}$, nor do we have a conceptual explanation for why these distributions take these forms.

\section{References}

[BS02] Andrei N. Borodin and Paavo Salminen. Handbook of Brownian Motion-Facts and Formulae. Probability and its Applications. Birkhäuser Verlag, Basel, 2nd edition, 2002. MR1912205 (2003g:60001)

[Car07] John Cardy. ADE and SLE. J. Phys. A, 40(7):1427-1438, 2007, arXiv:math-ph/0610030. MR2303259 (2008k:82023)

[CN06] Federico Camia and Charles M. Newman. Two-dimensional critical percolation: the full scaling limit. Comm. Math. Phys., 268(1):1-38, 2006, arXiv:math.PR/0605035. MR2249794 (2007m:82032)

[CN07] Federico Camia and Charles M. Newman. Critical percolation exploration path and $\mathrm{SLE}_{6}$ : a proof of convergence. Probab. Theory Related Fields, 139(3-4):473519, 2007, arXiv:math/0604487. MR2322705 (2008k:82040)

[CT62] Z. Ciesielski and S. J. Taylor. First passage times and sojourn times for Brownian motion in space and the exact Hausdorff measure of the sample path. Trans. Amer. Math. Soc., 103(3):434-450, 1962. MR0143257 (26 \#816)

[CZ03] John Cardy and Robert M. Ziff. Exact results for the universal area distribution of clusters in percolation, Ising, and Potts models. J. Statist. Phys., 110(1-2):133, 2003, arXiv:cond-mat/0205404. MR1966321 (2004m:82057)

[Dub05] Julien Dubédat, 2005. Personal communication.

[Dup90] Bertrand Duplantier. Exact fractal area of two-dimensional vesicles. Physical Review Letters, 64(4):493, 1990.

[EMOT53] Arthur Erdélyi, Wilhelm Magnus, Fritz Oberhettinger, and Francesco G. Tricomi. Higher Transcendental Functions. Vol. I. McGraw-Hill Book Company, 1953. Based, in part, on notes left by Harry Bateman. MR0058756 (15,419i) 
[FK72] C. M. Fortuin and P. W. Kasteleyn. On the random-cluster model. I. Introduction and relation to other models. Physica, 57:536-564, 1972. MR0359655 (50 \#12107)

[Gri06] Geoffrey Grimmett. The Random-Cluster Model, volume 333 of Grundlehren der Mathematischen Wissenschaften [Fundamental Principles of Mathematical Sciences]. Springer-Verlag, Berlin, 2006. MR2243761 (2007m:60295)

[KN04] Wouter Kager and Bernard Nienhuis. A guide to stochastic Löwner evolution and its applications. J. Statist. Phys., 115(5-6):1149-1229, 2004, arXiv:math-ph/0312056. MR2065722 (2005f:82037)

[KW04] Richard W. Kenyon and David B. Wilson. Conformal radii of loop models, 2004. Manuscript.

[LSW02] Gregory F. Lawler, Oded Schramm, and Wendelin Werner. One-arm exponent for critical 2D percolation. Electron. J. Probab., 7: Paper No. 2, 13 pp., 2002, arXiv:math.PR/0108211. MR1887622 (2002k:60204)

[RY99] Daniel Revuz and Marc Yor. Continuous Martingales and Brownian Motion, volume 293 of Grundlehren der Mathematischen Wissenschaften [Fundamental Principles of Mathematical Sciences]. Springer-Verlag, Berlin, third edition, 1999. MR1725357 (2000h:60050)

[Sch01] Oded Schramm. A percolation formula. Electron. Comm. Probab., 6:115-120 (electronic), 2001, arXiv:math.PR/0107096. MR1871700 (2002h:60227)

[She06] Scott Sheffield. Exploration trees and conformal loop ensembles, 2006, arXiv:math.PR/0609167. Duke Math. J., to appear.

[Smi01] Stanislav Smirnov. Critical percolation in the plane: conformal invariance, Cardy's formula, scaling limits. C. R. Acad. Sci. Paris Sér. I Math., 333(3):239244, 2001. MR1851632 (2002f:60193)

[SW08a] Scott Sheffield and Wendelin Werner. Conformal loop ensembles: Construction via loop-soups, 2008. In preparation.

[SW08b] Scott Sheffield and Wendelin Werner. Conformal loop ensembles: The Markovian characterization, 2008. In preparation. 